\title{
Copy-Move Forgery Detection Using Deep Learning
}

\author{
Chris Templeton \\ Computer Science \\ Universidad Virtual Latinoamericano \\ Venezuela
}

\author{
Awazuddin Shah \\ Computer Science \\ Universidad Virtual Latinoamericano \\ Venezuela
}

Jawad Khan

Computer Science

Universidad Virtual Latinoamericano

Venezuela

\begin{abstract}
Due to the number of image editing tools available online, image tampering has been easy to execute. The quality of these tools has led these tamperings to steer clear from the naked eye. One such tampering method is called the Copy-Move tampering where a region of the image is copied and pasted elsewhere in the image. We propose a method to deal with this. First, the image is broken to blocks using discrete cosine transform. Next, the dimensionality is reduced using the gaussian RBF kernel PCA. Finally, a new iterative interest point detector is proposed and the image is then sent as input to a $\mathrm{CNN}$ that predicts whether the image has been forged or not. The experimental results showed that the algorithm gave an excellent percentage of accuracy, outperforming state of the art methods.
\end{abstract}

Keywords - copy-move, forgery, random forest

\section{INTRODUCTION}

During recent times, image tampering has become extremely feasible due to the easy availability of picture editing software. Such software has made it remarkably easy to manipulate, modulate and modify an image's content while not reducing the quality of the image. These changes do not leave any idea of modification to a user and these images end up on the internet and shared even though it has been modified. Videos and images hold high a level of importance in today's world. They are extremely important in fields like forensics, medical imaging, sports, research publications etc. Forging in these fields can lead to a lot of problems. For example, crime scenes can be forged and can lead to lack of evidence or help the criminal to escape.

Image forgery can broadly be divided into 3 categories:
1) Image Splicing
2) Image retouching
3) Copy-Move forgery.

\section{Image Splicing}

Image splicing is a forgery method where two or more images are taken and combined together. This combination generates a new image called the splicing image. Figure (1) shows an example of this.
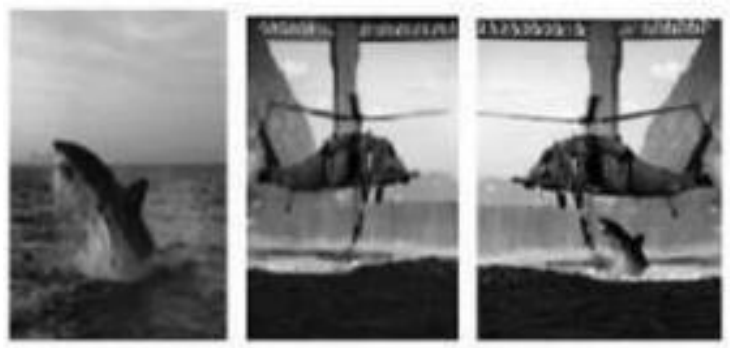

Figure 1. Image splicing combining two images

Image splicing forgery is hard to detect. This is because it is hard to find out the difference in either the region of the forgery or it's boundary.

\section{Image retouching}

Image retouching is another type of forgery. In this method subtle changes are made in the image by modulating changes in color, blur level etc. Another type of modulation is doing operations such as scaling, rotation etc to a part of an image and this creates a new image. Figure 2 shows an example of image retouching.
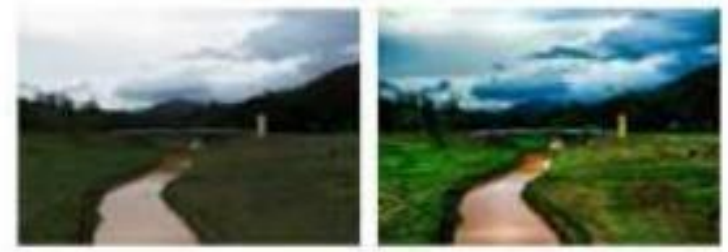

Figure 2. Image retouching example

\section{Copy-move forgery}

In this type of forgery a portion of the image is copied and pasted over another region to hide some information. Figure 3 shows an example of this type of forgery. 


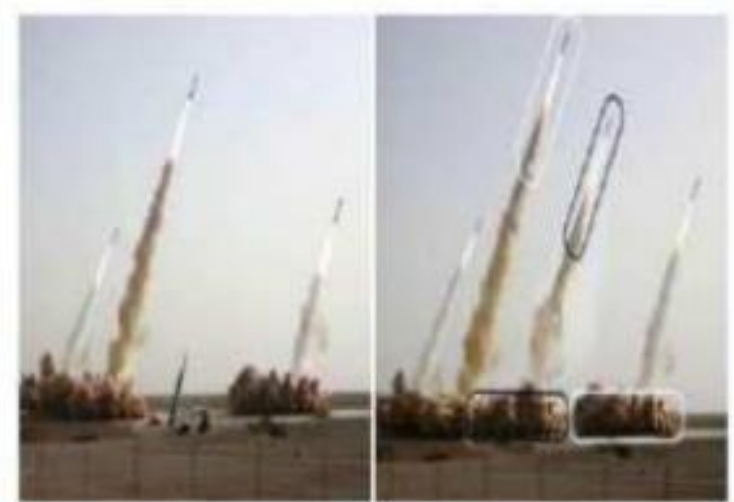

Figure 3. Copy-move forgery example

\section{RELATED WORK}

This paper proposes an approach for copy-move forgery detection.

In general there are two types of copy-move forgery detection algorithms: block-based or keypoint-based. Copymove forgery methods generally involve comparing regions of interest. This is done by counting a similarity score.

An example of how to count this similarity was proposed by Zhang et al [1].

Block based approaches extract blocks from the image and compare the similarities between blocks to determine blocks having high similarity.

Key-point based approaches would extract key points in the images as features and compare regions of interest using these key-points. Regions with high similarity were considered forged.

\section{A. Block Based Approaches}

Fridrich et al [2] proposed a method to detect forgery using Discrete Cosine Transfrom (DCT). Lexicographical sorting was done to reduce the cost of computation and pairs of neighboring blocks were considered as possibly forged. A similarity measure was calculated among the blocks. The drawback was an assumption made regarding a lack of postprocessing operations done.

An approach utilizing Discrete Wavelet Transform(DWT) and singular value decomposition(SVD) was proposed by Li et al [3]. They extracted low frequency coefficients by utilizing DWT. Next, they reduced the feature vector by using SVD.

Kang et al [4] proposed an enhancement of the same approach by using an advanced SVD. Myna et al [5] proposed an approach based on DWT and log-polar coordinates. The authors used DWT to obtain reduced dimension representation. The log-polar coordinates were used for mapping to sub-blocks of the image. Phase correlation and exhaustive searching was done to obtain matches between blocks.

Ryu et al [6] proposed an approach using Zernike moments. This algorithm was later on enhanced by Muhammad et al [7] by using an undecimated Wavelet Transform first. Both algorithms were invariant to roation, blurring, JPEG compression but they were still susceptible to scaling.
An approach using circular blocks were proposed by Cao et al [8]. DCT was used to extract DCT coefficients and then circular blocks were along with this to extract the features. This approach reduced the feature length and showed robustness against noise and blurring. However, the algorithm was still suspect to rotation, compression and scaling.

Another approach using circular blocks was proposed by $\mathrm{Li}$ et al [9]. In this, circular blocks were extracted and rotation invariant LBPs are applied to the blocks to extract the features. Next, a gaussian filter was used to obtain low frequency components. These are considered to be more stable for forgery detection. This method was not suited for forgeries with arbitrary angles.

\section{B. Keypoint based approaches}

Key-point based approaches eliminate the block division in the preprocessing stage of the algorithm itself. Distinctive features such as blobs, corners, edges etc are extracted using key points. A descriptor is then produced for a region around the features developed using the key-points. These regions are then used for the comparison to extract the similarity score.

Pun et al [10] proposed an approach where feature matching was done using Coefficient maps and thresholding. Pre-processing was done using DWT and segmentation.

Li et al [11] proposed a segmentation based approach to detect forgery. In this, small independent patches were extracted and the matching was done utilizing a transform matrix.

A method utilizing BSFN clustering was proposed by Liu et al [12]. The algorithm did not use any preprocessing method and instead utilized pixel sized regions of interest and then clustered them using BFSN. The algorithm showed robustness against scaling and rotation.

Keum et al [13] proposed a method to detect forgery using SIFT that was proposed by Lowe et al [14] as the method for key-point extraction. The regions were divided using mean shift clustering. The matching of regions were done using pattern entropy.

A method was proposed by Hashmi et al [15] wherein DWT was used to preprocess the image, key points were then obtained using SIFT and comparison of regions were done using the feature vectors. This method was robust to rotation and scaling. Jaberi et al [16] proposed a method to detect forgery by using a mirror reflection invariant feature descriptor proposed by Guo et al [17]. Region matching was done using RANSAC algorithm proposed by Fischler et al [18] and hysteresis thresholding

\section{PROPOSED APPROACH}

\section{A. Datasets}

We use two popular databases to aid us with our experimental accuracy. First, we use the IMD database proposed by Christlein in [19] and then with the CoMoFoD database proposed by Tralic et al [20]. In total, we used 2000 images for training using the small image category of CoMoFoD and testing was done on 300 images. 


\section{B. Proposed Approach}

We propose a novel method to detect copy-move forgery. First, the image is subject to Discrete Cosine Transform (DCT) to obtain blocks in the image. Next, we use a Gaussian Radial Basis Function (RBF) kernel Principal Component Analysis (PCA) to reduce dimensionality. We then propose and use a novel iterative interest point detector and then the image is passed to a $\mathrm{CNN}$ that predicts if the image has been forged or not.

C. Breaking image to blocks and dimensionality reduction

First we convert the image 'I' from color to grayscale and this is done as shown in (1).

$$
I=0.229 R+0.587 G+0.114 B
$$

Once the image is converted into grayscale we use a window of size $\mathrm{h} \mathrm{x} \mathrm{w}$ where ' $h$ ' is the height and ' $w$ ' is the width of the window to slide one pixel along the image from the top left to the bottom right. This is done to obtain overlapping blocks. A block is expressed as Brc, where ' $r$ ' is the block's row and ' $c$ ' is the block's column. Hence, the image I can be broken down to $\mathrm{N}$ overlapping blocks as shown in (2).

$$
N=(H-h+1) x(W-w+1)
$$

Next, to extract features from a block $\operatorname{Brc}(\mathrm{x}, \mathrm{y})$ of size $\mathrm{h} \mathrm{x}$ $\mathrm{w}$, where $\mathrm{x}, \mathrm{y}$ range from 0 to $\mathrm{N}-1$ we decompose the block using 2-D DCT function. The result is produced and stored as coefficients matrix $C(p, q)$ of size $h \mathrm{x}$ w that contains DCT coefficients.

We then use a Gaussian kernel function to transform C. The function applied is shown in (3).To this, we calculate two texture features as smoothness.

$$
K\left(x_{i}, x_{j}\right)=e^{-\frac{\left\|x_{i}-x_{j}\right\|^{2}}{2 \sigma^{2}}}
$$

The unknown in the equation is the Gaussian parameter. Applying this to $\mathrm{C}$ we obtain a new matrix called $\mathrm{M}$. The dimensionality is reduced to ' $n$ ' by using (4).

$$
1-\varepsilon=\frac{\sum_{z=1}^{n} \lambda_{c}}{\sum_{z=1}^{b} \lambda_{c}}
$$

\section{Iterative Interest point detector}

We propose a novel interest point detector. An adaptive approach is proposed. The interest point density is automatically adjusted over the image to concentrate more on the suspected regions of interest.

Numerous parts of an image may end up being considered as similar due to the self-similarity of natural images.

This happens generally in low-entropy areas. Probability of false matching is high in regions of low uniqueness. Due to this, we selected blocks that contained unique areas that are less likely to match.

We use $\mathrm{U}$ to represent the uniqueness of the ith point, $\mathrm{J}$ as the capacity of a point, $\mathrm{Y}$ as the certainty level, $\mathrm{X}$ as the set of interest points and $\mathrm{W}$ as the matching table. We define the uniqueness metric $U$ in (5).

This is calculated for each pixel in the block. The proposed equation was calculated based on experimental analysis.

$$
U_{i}=\frac{1}{B_{r, c}} \sqrt{\sum_{x=0}^{c} \sum_{y=0}^{r}\left(\Delta^{2} I(x, y)\right)^{2}}
$$

Each pixel is given a certainty value $\mathrm{Yi}$. This was calculated after reducing the dimensions of the image in a pyramid fashion.

The dimensions were iteratively reduced to 0.9 of the previous image starting with the original image until the image could no longer be reduced. We then defined a capacity level based on this certainty value called Ji. This was calculated as shown in (6).

$$
J_{i}=Y_{i} U_{i}
$$

To then define a point as an interest point, we compare it to the local density value di. If $\mathbf{J}$ is greater than $\mathrm{d}$ for $\mathrm{i}$ then the point is considered an interest point. The local density $\mathrm{d}$ is defined by a Gaussian kernel as shown in (7).

$$
d_{i}=\sum_{c, v=0}^{c=r, v=c} e^{\frac{\left(x_{c}, y_{v}\right)-J_{i}}{2 \sigma^{2}}}
$$

These input points are plotted in the image and sent as input to a $\mathrm{CNN}$ that predicts if the image has any forgery present.

During the training phase, the coefficients if the convolutional filter are learned automatically. These filters are represented as wij. This alternately uses a feedforward and backpropagation algorithm for passes of the image. The loss function is defined in (8).

$$
E=\frac{1}{m} \sum_{i=1}^{t} \sum_{k=1}^{c} y_{i}^{*(k)} \log \left(y_{i}^{(k)}\right)
$$

Here, $\mathrm{t}$ represents the number of training images and $\mathrm{c}$ is the number of output neurons. The * represents the true label. We then use the stochastic gradient descent to train the model. We iteratively update the kernel coefficients during the backpropogration passes using the rule shown in (9) and (10). 


$$
\begin{gathered}
w_{i j}^{(n)}=w_{i j}^{(n)}+\Delta w_{i j}^{(n)} \\
\Delta w_{i j}^{(n)}=m \cdot \Delta w_{i j}^{(n)}-\text { d. } \epsilon \cdot w_{i j}^{(n)}-\epsilon \cdot \frac{\delta E}{\delta w_{i j}^{(n)}}
\end{gathered}
$$

Here, $\mathrm{i}$ refers to the channel and $\mathrm{j}$ refers to the kernel matrix hidden layer that convolves with the i channel. ' $m$ ' and 'd' refer to momentum and decay and are used for fast convergence as proposed by LeCun et al [25].

The CNN classifies the output as either forged or not forged. The architecture of the proposed approach is shown in Figure 4.

\section{EXPERIMENTAL ANALYSIS}

The proposed method was tested and compared using first the IMD and CoMoD databases. The proposed method was compared against the method proposed by Huang et al [24], Cao et al [8] along with SIFT, SIFT-HOG and SURF.

Table 1 corresponds to the accuracy comparison for the algorithms tested using IMD database under rotation attacks. Table 2 corresponds to the accuracy comparison using IMD database with additive noise. Table 3 corresponds to the accuracy comparison using IMD database with JPEG compression. Table 1 corresponds to the accuracy comparison for the algorithms tested using CoMoD database under rotation attacks.

Table 5 corresponds to the accuracy comparison using CoMoD database with additive noise. Table 6 corresponds to the accuracy comparison using CoMoD database with JPEG compression.

\begin{tabular}{|l|c|}
\hline Method & Accuracy \\
\hline \hline SIFT & 88.64 \\
SURF & 87.48 \\
SIFT-HOG & 91.51 \\
Huang et al [24] & 95.43 \\
Cao et al [8] & 94.81 \\
Proposed & 99.48 \\
\hline
\end{tabular}

Table 1. Accuracy comparison using IMD rotation

\begin{tabular}{|l|c|}
\hline Method & Accuracy \\
\hline \hline SIFT & 85.45 \\
SURF & 86.01 \\
SIFT-HOG & 88.74 \\
Huang et al [24] & 96.83 \\
Cao et al [8] & 96.91 \\
Proposed & 98.89 \\
\hline
\end{tabular}

Table 2. Accuracy comparison using IMD with noise

\begin{tabular}{|l|c|}
\hline Method & Accuracy \\
\hline \hline SIFT & 86.36 \\
SURF & 86.45 \\
SIFT-HOG & 87.78 \\
Huang et al [24] & 95.48 \\
Cao et al [8] & 95.37 \\
Proposed & 98.65 \\
\hline
\end{tabular}

Table 3. Accuracy comparison using IMD with JPEG compression

\begin{tabular}{|l|c|}
\hline Method & Accuracy \\
\hline \hline SIFT & 88.42 \\
SURF & 87.78 \\
SIFT-HOG & 91.61 \\
Huang et al [24] & 95.31 \\
Cao et al [8] & 94.67 \\
Proposed & 98.31 \\
\hline
\end{tabular}

Table 4. Accuracy comparison using $\mathrm{CoMoD}$ rotation

\begin{tabular}{|l|c|}
\hline Method & Accuracy \\
\hline \hline SIFT & 86.15 \\
SURF & 86.41 \\
SIFT-HOG & 88.14 \\
Huang et al [24] & 97.13 \\
Cao et al [8] & 96.94 \\
Proposed & 99.17 \\
\hline
\end{tabular}

Table 5. Accuracy comparison using CoMoD with noise

\begin{tabular}{|l|c|}
\hline Method & Accuracy \\
\hline \hline SIFT & 84.51 \\
SURF & 86.16 \\
SIFT-HOG & 87.59 \\
Huang et al [24] & 96.03 \\
Cao et al [8] & 96.57 \\
Proposed & 98.71 \\
\hline
\end{tabular}

Table 6. Accuracy comparison using IMD with JPEG compression

\section{CONCLUSION}

A method to detect Copy-Move forgery was proposed. First the image is subject to ACC and feature vector obtained is passed as input to a RBF-SVM and a prediction score is calculated.

Next, the image is subject to NCC and the feature vector is passed as input to a linear regression model to obtain an entropy value as output. Both the outputs obtained are passed as input to a random forest classifier that classifies the image to two classes: forged or not forged.

The proposed method showed great accuracy, much better than older methods and slightly better than recent state of the art methods. Novelty was in the ensemble of the algorithms and in the calculation of the entropy. 


\section{References}

[1] Gowda, S.N. and Yuan, C., 2018, December. ColorNet: Investigating the importance of color spaces for image classification. In Asian Conference on Computer Vision (pp. 581-596). Springer, Cham.

[2] Fridrich, A.J., Soukal, B.D. and Luk, A.J., 2003. Detection of copy- move forgery in digital images. In in Proceedings of Digital Forensic Research Workshop.

[3] Kang, L. and Cheng, X.P., 2010, October. Copymove forgery detection in digital image. In Image and Signal Processing (CISP), 2010 3rd International Congress on (Vol. 5, pp. 24192421). IEEE.

[4] Myna, A.N., Venkateshmurthy, M.G. and Patil, C.G., 2007 December. Detection of region duplication forgery in digital images using wavelets and log-polar mapping. In Conference on Computational Intelligence and Multimedia Applications, 2007. International Conference on (Vol. 3, pp. 371-377). IEEE.

[5] Muhammad, G. and Hossain, M.S., 2011, August. Robust copymove image forgery detection using undecimated wavelets and Zernike moments. In Proceedings of the Third International Conference on Internet Multimedia Computing and Service (pp. 95-98). ACM.

[6] Cao, Y., Gao, T., Fan, L. and Yang, Q., 2012. A robust detection algorithm for copy-move forgery in digital images. Forensic science international, 214(1), pp.33-43.

[7] Li, L., Li, S., Zhu, H., Chu, S.C., Roddick, J.F. and Pan, J.S 2013. An efficient scheme for detecting copymove forged images by local binary patterns. Journal of Information Hiding and Multimedia Signal Processing, 4(1), pp.46-56.

[8] Pun, C.M., Yuan, X.C. and Bi, X.L., 2015. Image forgery detection using adaptive oversegmentation and feature point matching. IEEE Transactions on Information Forensics and Security, 10(8), pp.1705- 1716.

[9] Li, J., Li, X., Yang, B. and Sun, X., 2015. Segmentation-based image copy-move forgery detection scheme. IEEE Transactions on Information Forensics and Security, 10(3), pp.507-518.

[10] Gowda, S.N., 2017. Human activity recognition using combinatorial Deep Belief Networks. In Proceedings of the IEEE Conference on Computer Vision and Pattern Recognition Workshops (pp. 1-6). Gowda, S.N., 2017. Human activity recognition using combinatorial Deep Belief Networks. In Proceedings of the IEEE Conference on Computer Vision and Pattern Recognition Workshops (pp. 1-6).

[11] Keum, J.S., Lee, H.S. and Hagiwara, M., 2012, November. Mean shift-based SIFT keypoint filtering for region-of-interest determination. In Soft Computing and Intelligent Systems (SCIS) and 13th International Symposium on Advanced Intelligent Systems (ISIS), 2012 Joint 6th International Conference on (pp. 266-271). IEEE

[12] Gowda, S.N., 2016, September. Innovative enhancement of the Caesar cipher algorithm for cryptography. In 2016 2nd International Conference on Advances in Computing, Communication, \& Automation (ICACCA)(Fall) (pp. 1-4). IEEE

[13] Lowe, D.G., 1999. Object recognition from local scale-invariant features. In Computer vision, 1999. The proceedings of the seventh IEEE international conference on (Vol. 2, pp. 11501157). Ieee.

[14] Hashmi, M.F., Hambarde, A.R. and Keskar, A.G., 2013, December. Copy move forgery detection using DWT and SIFT features. In Intelligent Systems Design and Applications (ISDA), 2013 13th International Conference on (pp. 188-193). IEEE.

[15] Gowda, S.N., 2016, October. Using Blowfish encryption to enhance security feature of an image. In 2016 6th International Conference on Information Communication and Management (ICICM) (pp. 126129). IEEE

[16] Jaberi, M., Bebis, G., Hussain, M. and Muhammad, G., 2013, July. Improving the detection and localization of duplicated regions in copy-move image forgery. In Digital Signal Processing (DSP), 2013 18th International Conference on (pp. 1-6). IEEE

[17] Guo, X., Cao, X., Zhang, J. and Li, X., 2010. Mift: A mirror reflection invariant feature descriptor. Computer VisionACCV 2009, pp.536545.

[18] Gowda, S.N. and Yuan, C., 2020. StegColNet: Steganalysis based on an ensemble colorspace approach. arXiv preprint arXiv:2002.02413.

[19] Fischler, M.A. and Bolles, R.C., 1981. Random sample consensus: a paradigm for model fitting with applications to image analysis and automated cartography. Communications of the ACM, 24(6), pp.381395.

[20] Christlein, V., Riess, C., Jordan, J., Riess, C. and Angelopoulou, E.,
2012. An evaluation of popular copymove forgery detection approaches. IEEE Transactions on information forensics and security, 7(6), pp.1841 1854 .

[21] Gowda, S.N. and Sulakhe, S., 2016, April. Block based least significant bit algorithm for image steganography. In Proceedings of the Annual International Conference on Intelligent Computing, Computer Science \& Information Systems, Pattaya (pp. 16-19).

[22] Tralic, D., Zupancic, I., Grgic, S. and Grgic, M., 2013, September. CoMoFoDNew database for copy-move forgery detection. In ELMAR, 2013 55th international symposium (pp. 49-54). IEEE.

[23] Gowda, S.N., 2017, July. An intelligent fibonacci approach to image steganography. In 2017 IEEE Region 10 Symposium (TENSYMP) (pp. 1-4). IEEE.

[24] Lee Rodgers, J. and Nicewander, W.A., 1988. Thirteen ways to look at the correlation coefficient. The American Statistician, 42(1), pp.5966.

[25] Gowda, S.N., 2016, December. Advanced dual layered encryption for block based approach to image steganography. In 2016 International Conference on Computing, Analytics and Security Trends (CAST) (pp. 250-254). IEEE.

[26] Huang, Y., Lu, W., Sun, W. and Long, D., 2011. Improved DCTbased detection of copy-move forgery in images. Forensic science international, 206(1), pp.178-184.

[27] Gowda, S.N. and Vrishabh, D.N., 2017, April. A secure trigonometry based cryptography algorithm. In 2017 International Conference on Communication and Signal Processing (ICCSP) (pp. 0106-0109). IEEE. 